\title{
A Novel Time Decaying Approach to Obstacle Avoidance
}

\author{
Sankalp Arora and S. Indu \\ Faculty of Delhi College of Engineering, Delhi College of Engineering, Delhi University, \\ New Delhi-110042, India \\ ar.sankalp@gmail.com, s.induarediffmail.com
}

\begin{abstract}
One of the basic issues in navigation of mobile robots is the obstacle avoidance task which is commonly achieved using reactive control paradigm where a local mapping from perceived states to actions is acquired. The algorithms of this class suffer from a major drawback of exhibiting cyclic behavior when encountered with certain obstacle configurations. This paper presents a cognitive time decaying approach to overcome this cyclic behavior. The Dynamic Window algorithm is taken as an example for implementing this approach. To build a dynamic window based obstacle avoider, we use time decaying heuristic function for history mapping - which innately eliminates local minima even for a cluttered environment and gives the robot an exploratory nature best suited for map building purposes. The algorithm is successfully tested on a simulation, where it is shown to avoid the $\mathrm{U}$ bend problem of local minima.
\end{abstract}

Keywords: obstacle avoidance, dynamic window, local minima, cognitive.

\section{Introduction}

Many mobile robot systems combine a global path-planning module with a local obstacle avoidance module to perform navigation. While the global path planner determines a suitable path based on a map of the environment, the obstacle avoidance algorithm determines a suitable direction of motion based on recent sensor data. Obstacle avoidance is performed locally in order to ensure that real-time constraints are satisfied. A fast update rate allows the robot to safely travel at high speeds. Obstacle avoidance paradigm was introduced by Khatib in 1986 [1] through the concept of artificial potential fields. There were several reactive algorithms suggested [2], [3], [4] after that but none of them took the complete dynamics of the platform involved in their world models. In 1997 though Dieter Fox, Wolfram Burgard and Sebastian Thrun introduced the dynamic window approach [5] to obstacle avoidance. It differs from previous approaches by searching the velocity space for the appropriate controlling command. This is done by reducing the search space to the dynamic window which consists of velocities reachable in a short interval of time.

Although the dynamic window method has been very successful and is extensively in use, it suffers from the local minima problem that is getting stuck in cyclic behavior for certain configurations of obstacles. To avoid this cyclic behavior Global Dynamic Window Approach [6] was suggested by O. Brock and O. Khatib in 1999. It 
used a continuous navigation function with only one absolute minimum at the goal. This algorithm also had tendency to somehow move away from the goal for certain obstacle configurations as pointed out by Petter Ögren and Naomi Ehrich Leonard in the paper "A Convergent Dynamic Window approach to Obstacle Avoidance"[7] in 2005. The work of Petter Ögren and Naomi Ehrich Leonard suggests model predictive control method and lyapunov function based approach to resolve the issues of local minima and map trotting. Both the above works assume that the simultaneous localization and mapping problem is solved. Though this is possible using the range of SLAM techniques available but these algorithms are processor intensive and compromise the reactive nature of the algorithm.

In this paper, inspiration is drawn from the cognitive behavior of history mapping shown by humans in order to plan paths in an unknown environment. To implement the same, time decay based approach is suggested, to avoid cyclic behavior in obstacle avoiding algorithms. This approach considers and maps the recent past activities of the robot in a small local map and leaves time decaying stamps on its trail, when considering future paths these time decaying stamps are taken into account and path which is least traversed as well as more suited for reaching the goal is chosen. The tendency of the robot to take the least traversed path makes sure that it does not exhibit cyclic behavior. The flexibility of the approach lies in the fact that the increasing localization error can be dealt with by increasing the rate of decay of the time stamps and hence the approach can be implemented with or without using SLAM techniques.Therefore retaining the reactive nature of the original algorithm.

\section{Local Obstacle Avoidance - Dynamic Window Approach}

The dynamic window approach originally proposed is especially designed to deal with the constraints imposed by limited velocities and accelerations, because it is derived directly from the motion dynamics of synchro-drive mobile robots. In a nutshell, the approach considers periodically only a short time interval when computing the next steering command to avoid the enormous complexity of the general motion planning problem. The approximation of trajectories during such a time interval by circular curvatures results in a two-dimensional search space of translational and rotational velocities. This search space is reduced to the admissible velocities allowing the robot to stop safely.

Due to the limited accelerations of the motors a further restriction is imposed on the velocities: the robot only considers velocities that can be reached within the next time interval. These velocities form the dynamic window which is centered around the current velocities of the robot in the velocity space. Among the admissible velocities within the dynamic window the combination of translational and rotational velocity is chosen by maximizing an objective function. The objective function includes a measure of progress towards a goal location, the forward velocity of the robot, and the distance to the next obstacle on the trajectory. By combining these, the robot treads to its desire to move fast towards the goal and its desire to ship around obstacles. 


\section{The Cognitive Time Decaying Approach}

It is observed that that when humans get stuck in complex obstacle configuration, without the knowledge of global maps, history mapping is innately used by them along with approximate map building techniques. As a general tendency, humans during maze solving are least likely to take back the path on which they have just traversed. The time decay approach proposes to introduce the same cognitive behavior in obstacle avoidance algorithms.

Above is achieved by leaving a trail of time decaying time stamps on the map, wherever the robot treads. This trail is then used as history model to inform the algorithm about how much time back the robot had visited a cell. Using this past information a time heuristic is introduced to motivate the robot to take the least visited or the least recently visited path while avoiding the obstacles and moving towards the goal as suggested by the algorithm it is applied on. As the robot has the tendency to take the least recently visited path, its probability of getting stuck at the local minima or exhibiting cyclic behavior is also highly reduced. It should be noted here that the rate of decay of time stamps can be controlled as per the localization errors of a given platform; hence this approach can be used with or without SLAM techniques.

It is understandable that updating the whole map in every iteration would be difficult, hence only the active area (area around the robot) is updated for decayed trail values. Thus retaining the reactive nature of the algorithm as well as making it less susceptible to cyclic behavior. It is important here to note that only the area of map which was being refreshed by new proximity sensor reading is being refreshed now, even though there is a complete map present in the memory. This makes the algorithm more memory intensive without having any considerable affects on the runtime.

\section{The Time Heuristic}

The time decaying approach introduces a new Time_function, added to the original heuristic, so that the algorithm knows about its recent past and moves towards the goal while circumventing collisions as fast as it can and also avoiding the recently taken paths so as to avoid entering into the local minima situations. The resulting algorithm might not give the best path towards its goal but is highly reliable in terms of its reaching the goal.

The time function for a given candidate path is calculated by summing the time objectives of each cell that a candidate path encounters.

\subsection{The Time Objective -}

The time objective of a given cell is calculated by inverting the subtraction of current time from the time stamp left on the cell earlier, whole raise to power N. The previously unvisited cells are given a zero time objective value.

$$
\mathrm{T} \_ \text {obj }(\mathrm{t})=[1 /(\text { current_time }- \text { cell_time_stamp })]^{\mathrm{N}}
$$




\subsection{The Time Function -}

The time_function for any given path is calculated by summing the time objectives of all the cells to be encountered in a given path and multiplying it with a normalizing value. Mathematically it is given as-

$$
\text { T_function }(\mathrm{t})=(-1)^{*} \psi^{*} \sum \mathrm{T} \_\mathrm{obj}(\mathrm{t})
$$

The time function is thus obtained for every probable path in the given dynamic window. This function is then added to the existing heuristic to allow the algorithm to take an informed decision, considering its past states as well. As can be observed that the time objective for a given cell is always positive and attains a maxima when the time stamp of the cell is very close to current time. The time function inverts this relationship between time stamps and time objective values by introducing an extra negative sign. This ensures that the time function is maximum( 0$)$, for a path with no visited cells in it and minimum for the path having most recently visited cells in it. As we try to maximize the total heuristic the paths with least recently visited cells are automatically given more priority than the others, thus resulting in behavior that avoids cyclicity innately.

\subsection{Variation with $\mathrm{N}-$}

$\mathrm{N}$ signifies the rate of decay of time_obj values with time for a given cell. As $\mathrm{N}$ increases the rate of decay increases exponentially. For situations where the positioning error of a robot is less and does not increase rapidly with distance traversed then small values of $\mathrm{N}$ are acceptable for efficient implementation of algorithm. But if the positioning error of the robot increases rapidly with distance traversed then large values of $\mathrm{N}$ are required for the algorithm to work efficiently.

In the second case the robot acquires more exploratory nature and hence might take more time to get out of the obstacle configuration.

\subsection{Variation with $\Psi$ -}

Psi signifies the normalizing weightage allotted to the property of the robot to distinguish between visited and non - visited paths, and choosing the most non-visited path more than other paths. Greater psi is kept, lesser is the probability of the robot to choose the visited path. Although increasing the value of psi to a great extent would lead to the robot only choosing the least visited path even if the other functions like heading and distance heavily favor other paths and hence may lead to the failure of the algorithm. It should be noted that the time function is introduced in order to help the algorithm to take an informed decision and should not be the only criterion based on which the next state is decided. The new heuristic after incorporating timedecaying approach is given by-

$$
\mathrm{G}=\alpha^{*} \text { heading }(\mathrm{v}, \mathrm{w})+\beta^{*} \operatorname{dist}(\mathrm{v}, \mathrm{w})+\gamma^{*} \text { velocity }(\mathrm{v}, \mathrm{w})+\text { Time_func }(\mathrm{t})
$$




\subsection{Parameter Settings}

Although the performance of the obstacle avoidance depends on the weighting parameters $\mathrm{N}$ and psi, it is stable against slight changes of their values. Without any exhaustive tuning of these parameters we found values of 1.5 and $25 \%$ of total weightage, for $\mathrm{N}$ and psi to give good results. The tendency of the robot to avoid the past taken paths is defined by the relative value of psi and the memory of the algorithm is a function of N. By choosing different values for parameters, global knowledge about the environment can be transferred to the local obstacle avoidance. While higher values produce good results in wide environments, smaller values are more appropriate in narrow and populated hallways. An improvement upon the parameter tuning approach would be - self learning of parameters during run time. But the stated technique may need extensive overhauling of the simple history model currently used.

\section{Results}

As can be seen from figure 1, the simple Dynamic Window Approach algorithm is exhibiting cyclic behavior inside the $U$ shape obstacle configuration but successfully circumvents the obstacle (Fig. 2), using the time decaying approach. The robot is continuously trying to move towards its goal while avoiding the past paths that it has taken. The value of $\mathrm{N}$ in this case was taken to be 1 and psi was used for normalization and hence was taken to be $25 \%$ of the total weightage. It is important here to mention that this approach is not only limited to dynamic window based obstacle avoiders but also to other reactive algorithms with minor changes according to their heuristics.

Figure 3 shows the path treaded by the robot under a more exploratory heuristic, the value of $\mathrm{N}$ in this case was taken to be 3 and value of psi was also increased to an optimal value as can be seen the robot circumvents the obstacle but is more inquisitive in nature, and explores more area before reaching its goal. It is this nature of the algorithm that makes it suitable for use in conjunction with mapping techniques for map building purposes. Otherwise if the robot is used only for goal reaching purposes, it works best in conjunction with a SLAM technique.

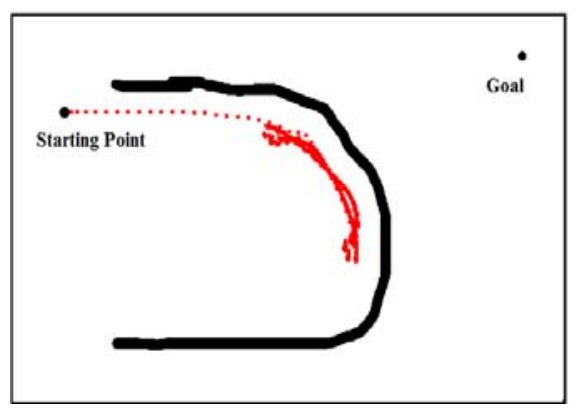

Fig. 1.

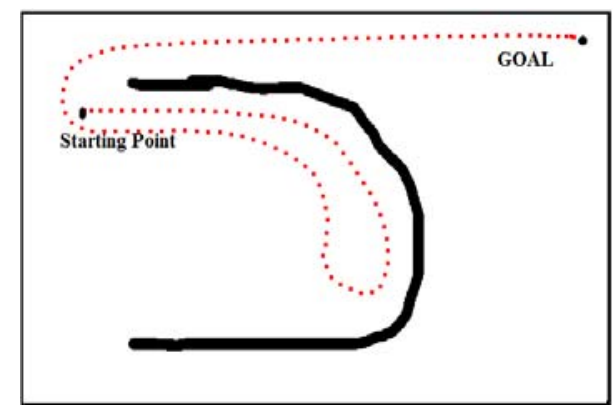

Fig. 2. 


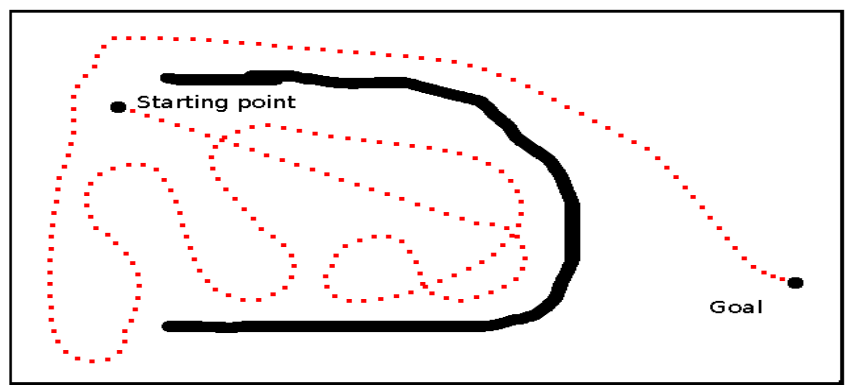

Fig. 3.

\section{Conclusion}

In this paper we first compare various obstacle avoidance techniques and then present the well established dynamic window approach in detail. We then presented a cognitive history mapping technique, achieved by leaving a trail of decaying time stamps along the robots past positions. As an example, the dynamic window approach was combined with time decaying methods, which has shown promising results in simulation. The approach is shown to innately avoid cyclic behavior without compromising on local and reactive nature of the algorithm. The algorithm is able to do so without considerably adding to the computation costs, whereas in other provably convergent approaches the addition on computation costs is high, and the algorithm shifts from local to global paradigm. The approach so presented is also shown to be flexible towards localization errors and gives an exploratory nature to the robot, best suitable for map building purposes. The time decaying approach can be combined with other obstacle avoidance algorithms as well with little changes in implementation details.

\section{References}

[1] Khatib, O.: Real-time obstacle avoidance for robot manipulator and mobile robots. The International Jour. of Rob. Res. 5(1), 90-98 (1986)

[2] Moravec, H.P.: Sensor fusion in certainty grids for mobile robots. AI Magazine, 61-74 (1988)

[3] Koren, Y., Borenstein, J.: Potential field methods and their inherent limitations for mobile robot navigation. In: Proc. IEEE Int. Conf. Robotics and Automation (April 1991)

[4] Borenstein, J., Koren, Y.: The vector field histogram - fast obstacle avoidance for mobile robots. IEEE Trans. Robot. and Auto. 7(3), 278-288 (1991)

[5] Fox, D., Burgard, W., Thrun, S.: The dynamic window approach to collision avoidance. IEEE Robot. Autom. Mag. 4, 23-33 (1997)

[6] Brock, O., Khatib, O.: High-speed navigation using the global dynamic window approach. In: Proc. IEEE Int. Conf. Robot. Autom., De troit, MI, May 1999, pp. 341-346 (1999)

[7] Ögren, P., Leonard, N.E.: A Convergent Dynamic Window approach to Obstacle Avoidance. IEEE Trans. Robot 21(2), 188-195 (2005) 